\title{
Modeling Cascading Failures in the North American Power Grid
}

\author{
Ryan Kinney ${ }^{1}$, Paolo Crucitti ${ }^{2}$, Réka Albert $^{3}$ and Vito Latora ${ }^{4}$ \\ 1. Department of Physics, University of Missouri-Rolla, MO 65409 \\ 2. Scuola Superiore di Catania, 95123 Catania, Italy \\ 3. Department of Physics and Huck Institutes of Life Sciences, \\ Pennsylvania State University, University Park, PA 16802 and \\ 4. Dipartimento di Fisica ed Astronomia, Universita' di Catania and INFN, 95124 Catania, Italy
}

\begin{abstract}
The North American power grid is one of the most complex technological networks, and its interconnectivity allows both for long-distance power transmission and for the propagation of disturbances. We model the power grid using its actual topology and plausible assumptions about the load and overload of transmission substations. Our results indicate that the loss of a single substation can lead to a $25 \%$ loss of transmission efficiency by triggering an overload cascade in the network. We systematically study the damage inflicted by the loss of single nodes, and find three universal behaviors, suggesting that $40 \%$ of the transmission substations lead to cascading failures when disrupted. While the loss of a single node can inflict substantial damage, subsequent removals have only incremental effects, in agreement with the topological resilience to less than $1 \%$ node loss.
\end{abstract}

PACS numbers:

As demonstrated in August of 2003, local disruptions of power distribution within the North American power grid can result in the loss of service to tens of millions of customers. At the heart of this vulnerability is the fact that the power grid has developed into one of the most complex and interconnected systems of our time, and the same capabilities that allow power to be transferred over hundreds of miles also enable the propagation of local failures into grid-wide events [1, 2]. To better understand such events, the power grid needs to be studied from a network perspective taking advantage of the recent advances in complex network theory [3].

Recently a great deal of attention has been devoted to the analysis of error and attack resilience of both artificially generated topologies and real world networks. The first approach that has been followed by researchers is that of static failures [4, 5, 6, 7, 8, 9, 10] and consists in removing a certain percentage of elements of the systems and evaluating how much the performance of the network is affected by the simulated failure. Following such an approach it has been shown that the removal of a sizable group of nodes can have important consequences. Nevertheless, in most real transportation/communication networks, the breakdown of a single or of a very small size group of elements can be sufficient to cause the entire systems to collapse, due to the dynamics of redistribution of flows on the networks. To take into account this phenomenon, dynamical approaches have been developed 11, 12, 13, 14, 15, 16. Those are based on the fact that the breakdown of a single component not only has direct consequences on the performance of the network, but also can cause an overload and consequently the partial or total breakdown of other components, thus generating a cascading effect.

Here, we use data on the network structure of the North American power grid obtained from the POWERmap mapping system developed by Platts, the energy information and market services unit of the McGrawHill Companies 21]. This mapping system contains information about every power plant, major substation, and $115-765 k \mathrm{~V}$ power line of the North American power grid. Our reconstructed network contains $N=$ 14, 099 substations and $K=19,657$ transmission (power) lines. The substations can be divided into three different groups: the generation substations set $G_{G}$, whose $N_{G}=$ 1,633 elements produce electric power to distribute, the transmission substations set $G_{T}$, whose $N_{T}=10,287$ elements transfer power along high voltage lines, and the distribution substations, whose $N_{D}=2,179$ elements distribute power to small, local grids.As previously observed, the North American power grid forms a connected network, thus in principle power from any generator is able to reach any distribution substation 10.

We model the power grid as a weighted 22, 23] graph $G$, with $N$ nodes (the substations) and $K$ edges (the transmission lines) and we represent it by the $N \times N$ adjacency matrix $\left\{e_{i j}\right\}$. The element $e_{i j}$ of such a matrix is 0 if there is no direct line from the substation $i$ to the substation $j$; otherwise it is a number in the range $(0,1]$ that represents the efficiency of the edge. Initially, for all existing edges, $e_{i j}$ is set equal to 1 , meaning that all the transmission lines are working perfectly.

Both in the static and in the dynamic approach, in order to quantify how well networks operate before and after the occurrence of breakdowns, a measure of performance has to be used. Here, as in 9, 14, 15], we use the average efficiency of the network 23 that, adapted to the case of the North American power grid, is defined as follows:

$$
E=\frac{1}{N_{G} N_{D}} \sum_{i \in G_{G}} \sum_{j \in G_{D}} \epsilon_{i j}
$$

where $\epsilon_{i j}$ is the efficiency of the most efficient path 
between the generator $i$ and the distribution substation $j$, calculated as the harmonic composition of the efficiencies of the component edges 24, 25, 26.

Once defined the efficiency as a measure of performance, the natural definition of the damage $D$ that a failure causes is the normalized efficiency loss [27]:

$$
D=\frac{E\left(G_{0}\right)-E\left(G_{f}\right)}{E\left(G_{0}\right)},
$$

where $E\left(G_{0}\right)$ is the efficiency of the network before the occurrence of any breakdown and $E\left(G_{f}\right)$ is the final efficiency that is reached by the system after the end of the transient due to a breakdown, i.e. when the network efficiency stabilizes.

In this paper we use the dynamical approach of the CML model of Ref. [14], adapting it to our network. We assume that each generator transfers power to all the distribution substations through the transmission lines. The generators have also transmitting capabilities, so they are both sources and intermediaries in power transmission. This scenario could seem unrealistic in the early days of electricity, when power was produced by local generators and transmitted only to the nearest distribution substations 2]. Nowadays, however, power is often redirected hundreds of kilometers away and our hypothesis that power from each generator can reach each distribution substation is not far from reality.

Adapting previous work on complex networks 17, 18] we define the load (also called betweenness) of each node with transmitting capabilities as the number of most efficient paths from generators to distribution substations that pass through the node. As in the CML model, we associate to each node $i$ a capacity $C_{i}$ directly proportional to the initial load $L_{i}$ it carries in the unperturbed network 11]:

$$
C_{i}=\alpha L_{i}(0) \quad i=1,2 . . N
$$

where $\alpha>1$ is the tolerance parameter that represents the ability of nodes to handle increased load thereby resisting perturbations.

If, due to external causes, a breakdown occurs at one or more nodes, so that they cannot work at all, the most efficient paths will change and the power/load, since it cannot be destroyed, will redistribute among the network. Sometimes this leads to a situation in which a certain number of nodes, forced to carry a load higher than their capacity, cannot function regularly anymore and show a degradation of their performance. Such a degradation can modify the most efficient paths, redistribute again the load on the network, and cause new nodes to be overloaded. If the overload caused by the initial breakdown is small, degradation will involve only a tiny part of the system, while if the overload to be reabsorbed is large enough, it will spread over the entire system in an avalanche mechanism, hindering any interaction among nodes. The degradation of performance is represented by the following dynamical model:

$$
e_{i j}(t+1)=\left\{\begin{array}{rll}
e_{i j}(0) / \frac{L_{i}(t)}{C_{i}} & \text { if } & L_{i}(t)>C_{i} \\
e_{i j}(0) & \text { if } & L_{i}(t) \leq C_{i}
\end{array}\right.
$$

where $j$ extends to all the first neighbors of $i$. In other words, when a node $i$ is congested, it is assumed that the efficiency of power transportation from(to) $i$ to(from) its first neighbors decreases linearly with the overload $L_{i}(t) / C_{i}$.

A benefit of the CLM model is that it does not assume that overloaded nodes fail irreversibly, but they have the possibility of working again if, by power rerouting, their load decreases below their capacity. In other words the effects of overload on nodes are reversible. Moreover, no explicit assumptions are made about the redistribution of loads, but this redistribution emerges naturally from the reorganization of efficient transmission paths following a node failure. Simulating a network failure involves removing a node from the network and monitoring the progression of overloading nodes. If the tolerance parameter $\alpha$ is high enough the network does not present the cascading effect typical of the redistribution of flows and its efficiency remains unaffected by the failure. If the tolerance parameter is very small, instead, the cascading effect takes place and the network rapidly collapses. For intermediate values of $\alpha$ the network degrades more slowly and its efficiency stabilizes to a value that is lower than the initial one. We observed that the efficiency of the network stabilizes into a steady state or small oscillations around an efficiency value in about 10-20 steps. (see inset of Figure [).

The reason for the occurrence of oscillations is strongly related to the reversibility of the effects of overload. Suppose that two paths exist from generator $i$ to the distribution substation $j$ (path $\mathrm{A}$ and path $\mathrm{B}$ ) and that under the condition of perfect functioning (i.e. before the occurrence of any breakdown) path $\mathrm{A}$ is more efficient than path $\mathrm{B}$. If at time $t$ some nodes of path $\mathrm{A}$ become overloaded, it may happen that B becomes the most efficient path from $i$ to $j$. If this implies that most of the load passing through $\mathrm{A}$ is redirected to $\mathrm{B}$, the nodes of the former path will recover efficiency to the detriment of some nodes of the latter one. Therefore the situation in which the most efficient path from $i$ to $j$ is A is restored and the redistribution of flows starts again its cycle. This switching between alternative paths causes the global efficiency to oscillate. Of course in a real world network the behavior is more complicated because the described cycle is concurrent with a redistribution of flows that involves the whole network. However the oscillations are evident all the same.

In our study, we have adopted two different types of node overload progression schemes. The first is single 
node removal in which a single node is removed at time zero and the network is progressed in time. This way, we can model the effects of an external perturbation of a single transmission node or generator. Nevertheless, it could happen that several nodes fail at the same time or in close succession or are shut down to save the equipment. In fact, it often happens that blackouts occurs because generators and transformers are hardwired to protect themselves in response to a drastic change. To model such type of cascading failure, we develop a second node overload progression scheme involving many cycles of node selection and removal and network progression.

In both the schemes, adopting the removal strategy from [14], we have chosen nodes either randomly (random removal) or selectively by highest load (load based removal) and once removed, the efficiency of the network and the load of the nodes were continually recalculated in time. Only generation and transmission substations were removed using the above strategy.

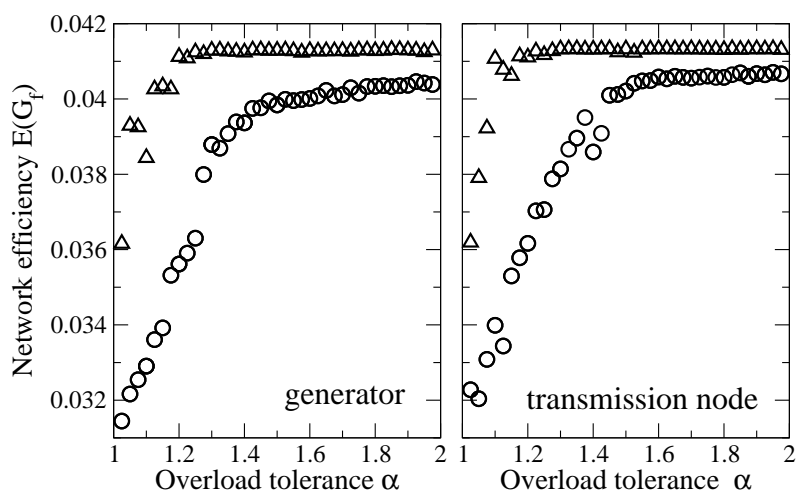

FIG. 1: Global efficiency of the power grid after the removal of random (triangles) or high-load (circles) generators or transmission substations. The unperturbed efficiency is $E\left(G_{0}\right)=0.04133$. As the overload tolerance $\alpha$ of the substations increases, the final efficiency approaches the unperturbed value. The random disruption curves were obtained by averaging over $10-100$ individual removals. The loadbased disruption curve is obtained by removing the highest load generator and transmission node, respectively.

Our first results use the single-node progression scheme for both removal types. Figure 1 shows a load based (circle) removal and an average of at least 10 random removals (triangle) for transmission and generation substations with final global efficiency as a function of the tolerance of the network. These figures indicate that above a critical tolerance value of approximately 1.42 , the removal of the highest loaded transmitter and generator substation has little effect on the overall network efficiency. However at values of tolerance below the critical value, the global efficiency can be reduced by over
20 percent. For random removals, the critical value is near 1.18 in both figures. These results clearly indicate that the loss of nodes with high load causes a higher damage in the system than the loss of random nodes. Moving beyond averages, Figures 2 present scatterplots of the efficiency of the network after the loss of randomly selected nodes for 40 different tolerance values. Two distinct trends are suggested from the efficiency versus tolerance scatterplot. The first, a horizontal line of points close to the unperturbed efficiency, indicates no efficiency loss for any tolerance level. The second, corresponding to tolerance-dependent damage, is a curve that initially increases linearly, then saturates at high tolerance levels.

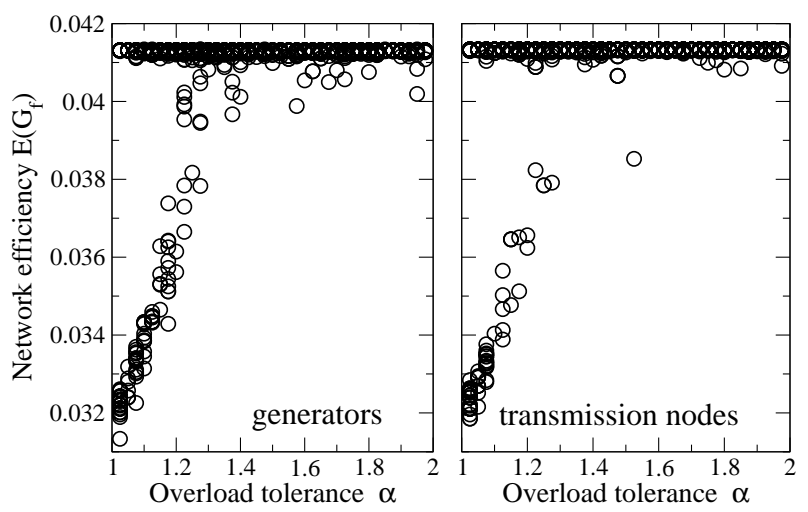

FIG. 2: Scatterplot of final network efficiency for given tolerance values for the removal of randomly selected nodes. A total of 1668 generator and 1558 transmission node removals are presented on this figure.

The scatterplot cannot illustrate the multiplicity of the observed (tolerance, efficiency) points. To gain insights into the distribution of efficiency loss we determine the cumulative damage distribution $P(D)$, i.e. the probability of observing damage larger than a given value $D$. Figure 3 shows the cumulative damage distribution for four tolerance values: $\alpha=1.025$ (circles), $\alpha=1.2$ (squares), $\alpha=1.4$ (diamonds) and $\alpha=1.8$ (triangles). As expected, the curves corresponding to distinct tolerance values have markedly different ranges, indicating that the higher the tolerance value the lower the probability to cause high damage. The long horizontal region of the $\alpha=1.025$ curve indicates a gap between high- and low damage, corresponding to the separation into two distinct damage behaviors observed in the scatterplot. However, the other distributions are relatively continuous, and all have power-law scaling regions with exponents whose magnitude increases with tolerance, varying between 0.5 and 2 . The probability distribution of disturbances on the power grid has been found to be a power law with exponent close to $-1.1[19,20]$, corresponding to 
an almost flat cumulative distribution. This is in closest agreement with our cumulative damage distribution for low tolerances, suggesting that the overload tolerance of the North American power grid is low.

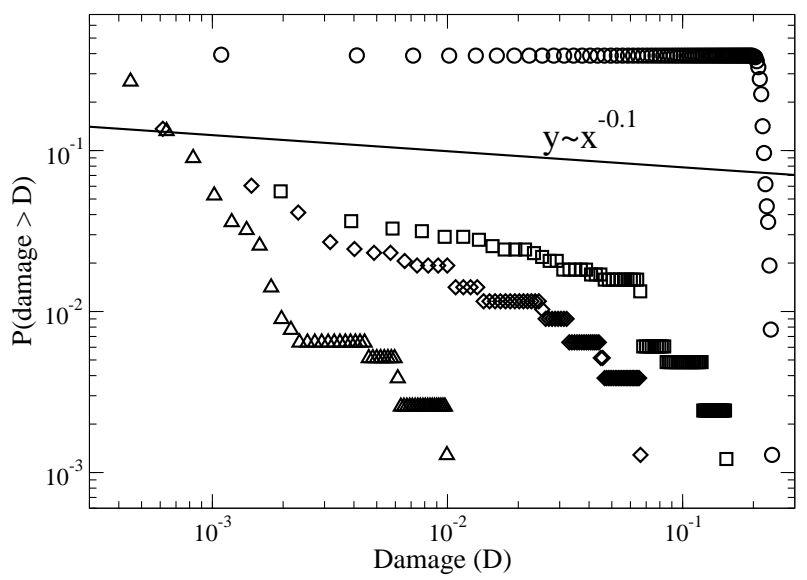

FIG. 3: Cumulative damage distribution for four different tolerance values, $\alpha=1.025$ (circles), $\alpha=1.2$ (squares), $\alpha=$ 1.4 (diamonds), and $\alpha=1.8$ (triangles). The continuous line indicates the cumulative distribution of disturbances on the power grid, i.e. $P(d>D)=D^{\delta-1}$, with $\delta \simeq 1.1[19,[20]$.

Comparing Figures 2 and 3 suggests the following question: do the two distinct (tolerance-dependent and independent) behaviors correspond to different classes of nodes? And if the answer is yes, what distinguishes the nodes in the two domains? To answer these questions we selected a sample of 15 nodes whose degrees and loads cover the entire range of degrees and loads, and studied the effect of their (separate) removal for a range of tolerance values. As Figure 4 shows, we find that some nodes' removal causes no decrease in network efficiency for the entire range of tolerance values. Therefore, the North American power grid is resilient to the loss of these nodes.

Included within the set of selected nodes is the node with the highest initial load. Interestingly, the removal of that particular node does not have the greatest effect upon the network. The node that has the greatest effect initially and a substantial effect over the entire range of tolerance values has roughly $80 \%$ the maximum load.

Based on Figure 4 we conclude that there are three separable classes of nodes:

1. Nodes whose removal causes no or very little damage for any tolerance. Around $60 \%$ of the nodes are in this category.

2. Nodes whose removal causes a tolerance-dependent

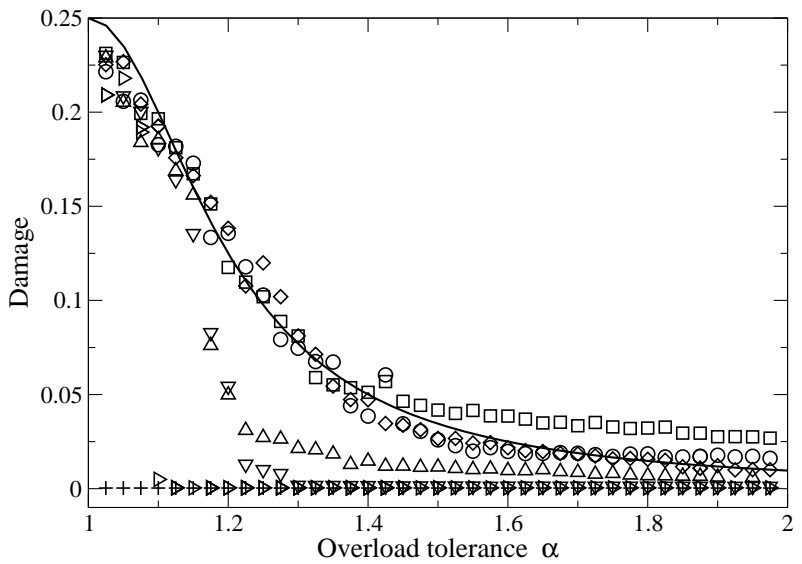

FIG. 4: Representative sample of node-dependent damage for different tolerance values. Two main types of behavior can be distinguished, one corresponding to no damage, and the other to a universal damage-versus-tolerance curve. A third type represents a discontinuous jump from tolerance-dependent to no-damage behavior. The continuous curve corresponds to Eq. $[5$

damage following the universal curve

$$
D=D_{0}\left(1-\frac{x^{\beta}}{K^{\beta}+x^{\beta}}\right)
$$

where $x=\alpha-1, D_{0}=0.25$ is the maximum damage, $K+1 \sim 1.2$ corresponds to the tolerance value causing half-maximal damage, and the exponent $\beta \simeq 2$.

3. Nodes that follow the tolerance-dependent curve for a while then suddenly jump to the no damage behavior.

The range of damage available at a given tolerance value is from zero (behavior 1) to the value given by the formula for behavior 2, in good agreement with the maximum damage indicated by Figure 3 .

We find that the nodes causing no efficiency loss (behavior 1) have both low betweenness and low degrees while the nodes that do affect the network upon removal have higher betweenness/degree. Figure 5(a) relates node degree and load with the damage caused by the node's removal for a set of 15 nodes. The plot indicates that, although there is no direct correlation between degree, load and efficiency loss, nodes that have both low degree and relatively low load will cause relatively little damage when perturbed. Figure 5(b) shows the load histogram of generators and transmission substations whose removal at tolerance $\alpha=1.025$ leads to 

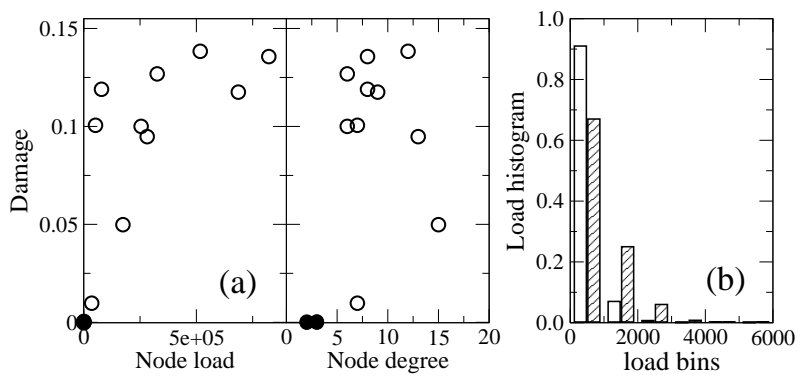

FIG. 5: (a) Correlation between node degree, load, and the efficiency loss its removal causes for 15 randomly selected nodes. The overload tolerance is $\alpha=1.2$. The nodes causing no damage (filled circles) have low loads and degrees.(b) Load histogram for the generators (white bars) and transmission substations (dashed bars) whose removal does not cause any damage at $\alpha=1.025$. A total of 639 generators and 476 transmission substations were included in this plot.

no efficiency loss. Each bin corresponds to a load range of 1000 . We find that $90 \%$ of no-damage-causing generators have loads $<1000$ and degree $<3$, while $90 \%$ of nondamage-causing transmission nodes have load $<2000$ and degree $=2$. The fraction of generators with degree 1 , expected to cause insignificant efficiency loss, is $72 \%$. Thus the network's resilience is higher than trivially expected.

Moving to the cascading failure, figure [6] shows a transmitter substation load based failure at a tolerance of $\alpha=1.025$. Here we remove the highest-load node, wait for the system to stabilize, then find and remove the current highest-load node, repeating this iteration several times. Interestingly, the final global efficiency after nearly thirty nodes removed is within ten percent of the final global efficiency for a single-node removal of same type and tolerance. The first node removed does the most damage while each successive removal does little to the worsening of efficiency. Similar behavior is recorded for generators. In random removals most behaviors, due to the higher probability of selecting a low degree and low betweenness node, reach stability, where the efficiency remains roughly constant, fter the first removal as in figure 6

This results is complementary and similar in spirit to the results of static transmission node removals [10] where the removal of up to $1 \%$ of the nodes had little effect on the connectivity of the power grid. As reference 10] has found, in this regime the connectivity of the grid, in other words the reachability between generators and distribution substations, decreases approximately proportionally with the fraction of nodes removed. Here we obtain efficiency loss (damage) of $40 \%$ after the removal of $0.33 \%$ of the high-load transmission nodes. Both of these results suggest that perturbations higher than $1 \%$ are needed for catastrophic failure.
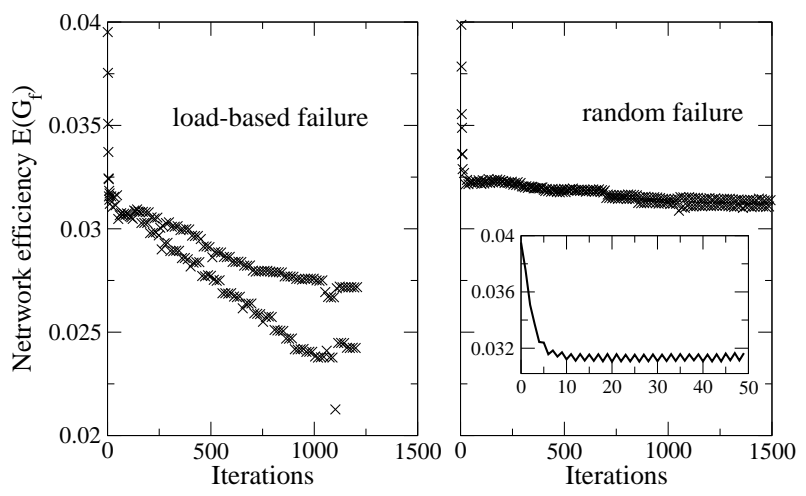

FIG. 6: Cascading failure with 30 consecutive node removals. A new node was removed at multiples of 50 iterations, the selection was either based on the highest load (left) or random (right). Inset: typical evolution of network efficiency after the removal of a single node.

These results are simultaneously reassuring and ominous. The North American power grid has been proven both theoretically and empirically to be highly robust to random failures. However, this research highlights the possible damage done to the network by a more targeted attack upon the few transmission substations with high betweenness and high degree. Our results, taken together with the observed disturbance distribution, suggest that even the loss of a single high-load and high-degree transmission substation reduces the efficiency of the power grid by $25 \%$. This vulnerability at the transmission level deserves serious consideration by government and business officials so that cost effective counter measures can be developed. Two possibilities include reducing the load upon the highly loaded nodes by building more transmission substations and controlling the spread of the cascade [28, 29], or by producing power on a more local level via environmentally friendly methods.

The authors wish to thank Gary L. Nakarado, Donna Heimiller and Steven Englebretson for their help in obtaining the POWERmap network data. The work of R. K. was supported by the Pennsylvania State University Research Experiences for Undergraduates program. R. A. gratefully acknowledges a Sloan Fellowship in Science and Engineering.

[1] Electricity Technology Roadmap, 1999 Summary and Synthesis, by the Electric Power Research Institute, http://www.epri.com/corporate/discover_epri/roadmap/

[2] A.-L. Barabási, The New York Times, August 16 (2003).

[3] R. Albert and A.-L. Barabási, Reviews of Modern Physics 74, 44-94 (2002); A.-L. Barabási Linked: The New Science of Networks (Perseus Publishing, Cambridge, 2002); D. J. Watts Six Degrees: The Science of a Connected Age (W. W. Norton \& Co., New York, 2003); S. N. Dorogovtsev and J. F. F. Mendes, Evolution of Net- 
works: From Biological Nets to the Internet and $W W W$ (Oxford University Press, Oxford, 2003); M. E. J. Newman, SIAM Review 45, 167 (2003).

[4] R. Albert, H. Jeong, and A.-L. Barabási, Nature 406, 378 (2000).

[5] R. Cohen, K. Erez, D. ben-Avraham, and S. Havlin, Phys. Rev. Lett. 85, 4626 (2000).

[6] D. S. Callaway, M. E. J. Newman, S. H. Strogatz, and D. J. Watts, Phys. Rev. Lett. 85, 5468 (2000).

[7] R. Cohen, K. Erez, D. ben-Avraham, S. Havlin, Phys.Rev. Lett. 86, 3682 (2001).

[8] A. E. Motter, T. Nishikawa, Y. Lai, Phys. Rev. E 66, 065103 (2002).

[9] P. Crucitti, V. Latora, M. Marchiori and A. Rapisarda, Physica A 320, 622 (2003).

[10] R. Albert, I. Albert and G.L. Nakarado, Phys. Rev. E 69, 025103(R) (2004).

[11] P. Holme and B. J. Kim, Phys. Rev. E 65, 066109 (2002).

[12] A. E. Motter and Y. Lai, Phys. Rev. E 66, 065102(R) (2002).

[13] Y. Moreno, R. Pastor-Satorras, A. Vásquez and A. Vespignani, Europhys. Lett. 62, 292 (2003).

[14] P. Crucitti, V. Latora, and M. Marchiori, Phys. Rev. E 69, 045104(R) (2004).

[15] P. Crucitti, V. Latora, M. Marchiori, Physica A 338, 92 (2004).

[16] I. Dobson, B. A. Carreras and D. E. Newman, in Proceedings of Hawaii International Conference on System Sciences, January 2003, Hawaii

[17] K.-I. Goh, B. Kahng and D. Kim, Phys. Rev. Lett. 87,
278701 (2001).

[18] M. E. J. Newman, Phys. Rev. E 64, 016132 (2001).

[19] B. A. Carreras, D. E. Newman, I. Dolrou and A. B. Poole, in Proceedings of Hawaii International Conference on System Sciences, January 2000, Maui, Hawaii.

[20] B. A. Carreras, V. E. Lynch, D. E. Newman, I. Dobson, in Proceedings of Hawaii International Conference on System Science, January 2003, Hawaii.

[21] Our access to this data was made possible by the National Renewable Energy Laboratory.

[22] S. Wasserman and K. Faust, Social Networks Analysis, Cambridge University Press, Cambridge, 1994.

[23] V. Latora and M. Marchiori, Phys. Rev. Lett. 87, 198701 (2001).

[24] J. Smith, Commun. ACM 31, 1202 (1988).

[25] R. Jain, The Art of Computer Systems Performance Analysis, Wiley, New York, 1991.

[26] The harmonic composition of $N$ numbers $x_{1}, x_{2}, \ldots, x_{N}$ is defined as $\left[\sum_{i}^{N} 1 / x_{i}\right]^{-1}$

[27] V. Latora, M. Marchiori, cond-mat/0407491(2004)

[28] A. E. Motter, Phys. Rev. Lett 93, 098701 (2004).

[29] Note that it is not possible to reduce the load of transmission substations by eliminating low-load (leaf) nodes. These nodes correspond to generators with one outgoing power line or distribution substations that have one incoming high-voltage power line. Consequently eliminating these nodes would diminish power generations or disconnect it from power consumption. 